\title{
Frequency Modulation Technique for Prosodic Modification
}

\author{
Jinfu $\mathrm{Ni}^{1,2}$, Shinsuke Sakai ${ }^{1,2}$, Tohru Shimizu ${ }^{1,2}$, and Satoshi Nakamura ${ }^{1,2}$, \\ ${ }^{1}$ National Institute of Information and Communications Technology, Kyoto \\ ${ }^{2}$ ATR Spoken Language Communication Research Laboratories, Kyoto
}

\begin{abstract}
Modulation of speaking tone in frequency can make speech interesting and convey subtle meaning in communication. We present a frequency modulation (FM) technique for prosodic modification to consider communicative speech synthesis. This technique provides a mathematical formulation for representing speaking tone and manipulating FM in a unified framework. Two experiments are conducted with a text-to-speech system to which a module of FM-based prosodic modification is added. One is to enhance emphasis in words when synthesizing Chinese conversational speech. The other is to modify readingstyle prosody while conveying good and bad news in Japanese; this is done by using the FM technique to shift the frequency ranges and rescale the fundamental frequency contours jointly. The experimental results indicated that the native speakers identified $90 \%$ of samples with emphases and $78 \%$ of "good news" as well as $94 \%$ of "bad news" samples. The FM technique is vital for making synthetic speech communicative.

Index Terms: frequency modulation, prosodic modification, intonation, speech synthesis
\end{abstract}

\section{Introduction}

A change in frequency in speaking tone provides the listener with a signal that something is happening. Modulation can thus be used to enhance emphasis in words, typically with rising and lowering tones. The term frequency modulation in this paper means to modulate speaking tone, focusing particularly on fundamental frequency $\left(F_{0}\right)$ contours, according to certain assigned adjusting proportions for speech synthesis.

Approaches based on hidden Markov models (HMM) [1] have been successfully used in modeling speech prosody, including phone duration, power, and $F_{0}$. Furthermore, significant progress has been made in corpus-based unit concatenative synthesis technology [2] [3]. These two things have led to an improvement in voice quality of synthetic speech, which in turn has led to it becoming more common. For example, it has been applied to speech-to-speech translation systems [4]. The problem is that for some applications, reading-style speech is no longer adequate because it lacks the aspects of communication, which are basically conveyed by speaking tone. In particular, reading-style prosody is far from satisfactory in most situations that involve human-machine dialogs or machine-mediated human-human dialogs. Therefore, there has been a lot of research on expressive and conversational speech synthesis so as to improve expressiveness of synthetic speech [2] [5] [6] [7].

In this paper we present a novel frequency modulation technique for prosodic modification in speech synthesis. Our motive for attempting prosodic modification is due to the fact that current text-to-speech (TTS) systems, such as XIMERA [3], already offer quite natural reading-style prosody. In an interactive dialog system, for example, it is desired to make synthetic speech more communicative. Thus a prosodic modification technique is necessary for modifying the reading-style prosody. The proposed frequency modulation technique will provide a unified framework for separately representing the speaking tone (i.e., the observed $F_{0}$ contours) and the adjusting proportions on one hand, and manipulating the modulation on the other. The rest of this paper is organized as follows. Section 2 presents our methodology. Section 3 demonstrates this technique within the framework of XIMERA whereby a module of prosodic modification is added for adding some expressive dimensions to synthetic speech. Section 4 discusses our method and conventional methods. Section 5 concludes this paper.

\section{Outline of the methodology}

\subsection{Resonance curves}

We consider frequency modulation in the light of using resonance curves to deal with the interaction of tone and intonation in [8]. A resonance curve in Eq (1), characterizing the amplifying rates of forced vibrations, is a function of the frequency ratio and the damping ratio of a forced vibrating system.

$$
A(\lambda, \zeta)=\frac{1}{\sqrt{\left(1-\left(1-2 \zeta^{2}\right) \lambda\right)^{2}+4 \zeta^{2}\left(1-2 \zeta^{2}\right) \lambda}},
$$

where $\lambda$ indicates the frequency ratio and $\zeta$ the damping ratio $\left(\zeta^{2}<0.5\right)$. As shown in the left panel of Fig. 1, $A(\lambda, \zeta)$ as a function of $\lambda$ shows a bell-shape pattern when given $\zeta$, while $\zeta$ functions to sharp or compress this bell-shape pattern. Furthermore, $A(1, \zeta)$ indicates the peaks of these patterns, and $A(0, \zeta)=A(2, \zeta)=1$, regardless values of $\zeta$. Equation (1) has been used to model $F_{0}$ contours as described in [9].

\subsection{Frequency modulation}

A change in frequency in speaking tone provides the listener with a signal that something is happening. Speaking tone is
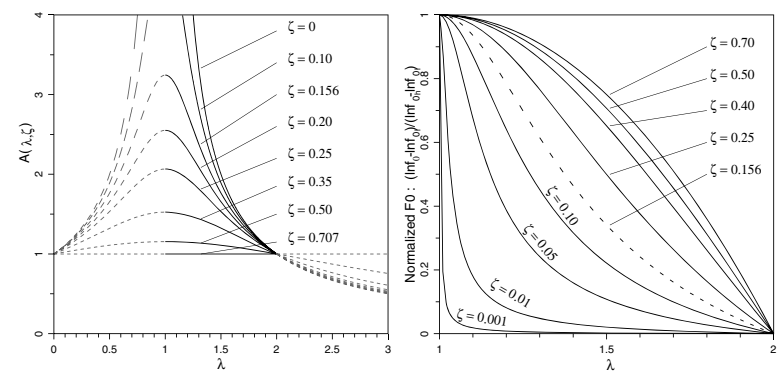

Figure 1: Resonance curve $A(\lambda, \zeta)$ (the left panel) and the warping functions between normalized $\log F_{0} \in[0,1]$ and $\lambda \in$ $[1,2]$ at several values of $\zeta$ (the right panel). 
manifested in utterances as a sequence of fundamental frequency. The voice range of a speaker in a speaking style can be characterized by a frequency range in $\mathrm{Hz}$, after this, denoted by $\left[f_{0_{l}}\right.$ (low boundary) and $f_{0_{h}}$ (high boundary)]. For any $f_{0} \in$ $\left[f_{0_{l}}, f_{0_{h}}\right]$, it can be mapped to $\lambda$ through the following nonlinear warping function.

$$
\frac{\ln f_{0}-\ln f_{0_{l}}}{\ln f_{0_{h}}-\ln f_{0_{l}}}=\frac{A(\lambda, \zeta)-A(2, \zeta)}{A(1, \zeta)-A(2, \zeta)},
$$

where $f_{0_{h}}$ is forcedly mapped to peak $A(1, \zeta)$ and $f_{0_{l}}$ to $A(2, \zeta)$, respectively. Use of a log scale was suggested in [10].

The right panel of Fig. 1 shows a set of warping functions, depending on the values of $\zeta$. It is clear that $\lambda \in[1,2]$ can be mapped to multiple $F_{0}$ values in $\left[f_{0_{l}}, f_{0_{h}}\right]$ when $\zeta$ is altered. This forms the basis of frequency modulation in that $\lambda$ and $\zeta$ can be used to represent the observed $F_{0}$ contours and the adjusting proportions, respectively. The Observed $F_{0}$ contours mean those synthesized by a TTS system, such as XIMERA [3].

\subsection{Representing observed $F_{0}$ contours (speaking tone)}

Let $\hat{F}_{0}(t)$ denote the observed $F_{0}$ contours within frequency range $\left[\hat{f}_{0_{l}}, \hat{f}_{0_{h}}\right]$ and $\hat{\Lambda}(t)$ represent the corresponding $\lambda$ values of $\hat{F}_{0}(t)$ according to Eq. (2), given $\zeta=\zeta_{0}$. After this, $\zeta_{0}=$ 0.156 (an empirical value). The warping function at $\zeta=\zeta_{0}$ is shown by the dashed lines on the right panel of Fig.1. In the mathematical term, the mapping relation between $\hat{\Lambda}(t)$ and $\hat{F}_{0}(t)$ can be rewritten as

$$
A\left(\hat{\Lambda}(t), \zeta_{0}\right)=\frac{\left[A\left(1, \zeta_{0}\right)-A\left(2, \zeta_{0}\right)\right] \ln \frac{\hat{F}_{0}(t)}{\hat{f}_{0_{l}}}}{\ln \hat{f}_{0_{h}}-\ln \hat{f}_{0_{l}}}+A\left(2, \zeta_{0}\right) .
$$

There is no analytical solution to $\hat{\Lambda}(t)$. However, it can be precisely estimated by an iteration procedure as described in [8].

\subsection{Representing adjusting proportions}

The adjusting proportions are represented by $\zeta$ as mentioned above. Let $Z(t)$ denote the adjusting proportions used for modulating $\hat{F}_{0}(t) . Z(t)$ shall be designed to convey specific communication functions. However, it is still an open question as how to automatically design $Z(t)$ for this purpose.

\subsection{Modulating observed $F_{0}$ contours}

Given $\hat{\Lambda}(t)$ (representing the observed $F_{0}$ contours in $\lambda$ ) and $Z(t)$ (the adjusting proportions), the resultant $F_{0}$ contours are computed by

$$
\ln F_{0}(t)=\frac{A(\hat{\Lambda}(t), Z(t))-A(2, Z(t))}{A(1, Z(t))-A(2, Z(t))} \ln \frac{f_{0_{h}}}{f_{0_{l}}}+\ln f_{0_{l}},
$$

where $F_{0}(t)$ stands for the resultant $F_{0}$ contours. Frequency range $\left[f_{0_{l}}, f_{0_{h}}\right]$ can be the original, i.e., $\left[\hat{f}_{0_{l}}, \hat{f}_{0_{h}}\right]$ for $\hat{F}_{0}(t)$, or a target range. When $\left[f_{0_{l}}, f_{0_{h}}\right]$ is different from $\left[\hat{f}_{0_{l}}, \hat{f}_{0_{h}}\right]$, frequency range shift is performed as well. In other words, this technique can shift frequency ranges and rescale $F_{0}$ contours jointly. Also, if $Z(t)=\zeta_{0}, f_{0_{l}}=\hat{f}_{0_{l}}$, and $f_{0_{h}}=\hat{f}_{0_{h}}$, then $F_{0}(t)$ $=\hat{F}_{0}(t)$, provided that there is no converting error.

\section{Experimental evaluation}

\subsection{Test bed: Adding prosodic modification to XIMERA}

We test the frequency modulation technique within the framework of XIMERA TTS system [3], which can synthesize

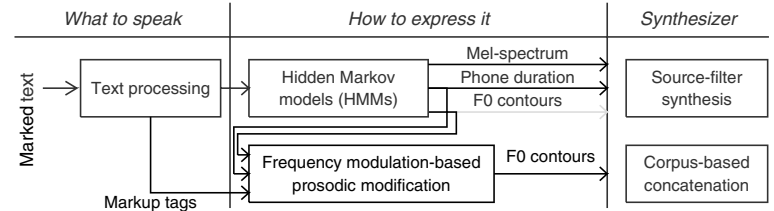

Figure 2: Schematic diagram of performing prosodic modification within the framework of TTS system XIMERA.

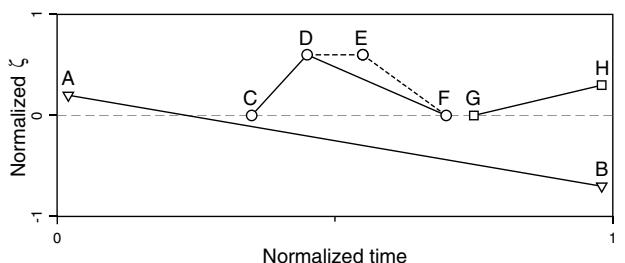

Figure 3: Schematic diagram of the basic patterns defined by tags baseline (line $A B)$, cap $(C D F / C D E F)$ and toend (line $G H$ ).

Japanese, Chinese, and English voices at present. To make a test bed, we reform XIMERA to add a frequency modulationbased module for prosodic modification as shown in Fig. 2. Thus the test bed consists of four modules: text processing, HMM-based prediction of speech parameters, prosodic modification, and synthesizers. The module of text processing extracts linguistic information from the input text, such as phrase dependency structure, the phrase length, and syllable position in a phrase, and processes the markup tags that are inserted in the input text. The makeup tags are used to specify the adjusting proportions for prosodic modification. The HMM module initially generates prosodic parameters and mel-cepstral coefficients as well [11]. The test bed has two types of speech synthesizer: source filter and corpus-based concatenation synthesis.

The module of prosodic modification performs frequency modulation of the HMM-generated $F_{0}$ contours according to the markup tags described below. The output $F_{0}$ contours of this module take place of the HMM-generated $F_{0}$ contours. They, together with the other speech parameters, are then used as the targets for unit-selection-based concatenative synthesis, or directly control the source filter to synthesize speech.

We define four markup tags, in which three tags are used to specify three piecewise-linear patterns, as shown in Fig. 3. These patterns and their combinations can describe frequently used intonation patterns, such as declination and rising/falling ending. The other is related to the frequency range shift. The four tags are described as follows.

- basline $[k, b]$ : Defining a base line $\zeta_{n}(t)=k \times t+b$ by two parameters $k$ and $b \in(-1,1)$, where $\zeta_{n}$ indicates normalized $\zeta$ in $[-1,1] ; \zeta_{0}$ is normalized to 0 .

- $\operatorname{cap}\left[\zeta_{n}^{c}\right]$ : Defining a cap pattern as $\mathrm{CDF} / \mathrm{CDEF}$ shown in Fig. 3. A parameter $\zeta_{n}^{c} \in[0,1]$ specifies its magnitude. The timings of points $\mathrm{C}, \mathrm{D}, \mathrm{E}$, and $\mathrm{F}$ are determined by a few rules, taking into account underlying linguistic and phonetic contexts where the tag is assigned.

- toend $\left[\zeta_{n}^{e}\right]$ : Defining a line GH from tag position $\mathrm{G}$ to the end of an utterance as shown in Fig. 3. The timing of point $\mathrm{G}$ is determined at which the tag is inserted. Parameter $\zeta_{n}^{e} \in[-1,1]$ specifies the adjusting proportion assigned at the end (i.e., $\mathrm{H}$ in Fig. 3).

- range $\left[\hat{f}_{0_{l}}, \hat{f}_{0_{h}}, f_{0_{l}}, f_{0_{h}}\right]$ : Specifying the original frequency range $\left[\hat{f}_{0_{l}}, \hat{f}_{0_{h}}\right]$ and a target range $\left[f_{0_{l}}, f_{0_{h}}\right]$. 
All of the basic patterns assigned for a sentence are added together to form piecewise-linear $Z_{n}(t) \in[-1,1] . Z_{n}(t)$ is then smoothed by a filter of moving average with a $25 \mathrm{~ms}$ window and further converted as $Z(t) \in[0,0.7]$ (i.e., $\zeta^{2}<0.5$ ). A conversion of $\zeta_{n}$ is defined as follows.

$$
\zeta= \begin{cases}\left(0.7-\zeta_{0}\right) \times \zeta_{n}+\zeta_{0}, & \text { for } \zeta_{n} \geq 0 \\ \zeta_{0} \times \zeta_{n}+\zeta_{0}, & \text { for } \zeta_{n}<0\end{cases}
$$

We conduct two experiments with this test bed to demonstrate the frequency modulation technique by adding extra meaning to synthetic speech. The first experiment is to enhance emphasis in words. The second is to shift the frequency ranges and rescale the $F_{0}$ contours jointly. The latter is usually used by human when changing emotional states and speaking styles.

\subsection{Experiment 1: Enhancing emphasis in words}

Emphasis is one of ways of adding extra meaning to some words of an utterance. We chose ten Chinese sentences from a few dialog turns and selected a lot of content words to make them emphasized when synthesizing speech. The average length was 6 syllables per sentence. The source-filter synthesizer was used in this experiment.

The procedure of prosodic modification consists of three components. First, generating reading prosody by the HMMbased acoustic models built in XIMERA. The $F_{0}$ contours are then converted to $\hat{\Lambda}(t)$ in Eq. (3). Second, generating adjusting proportion $Z(t)$ according to the assigned markup tags. $Z(t)=$ $\zeta_{0}$ when no markup tag is assigned. Finally, performing the prosodic modification as expressed in Eq. (4).

Figure 4 shows an example. A Chinese sentence "xian4 zai4ji3dian3le0?" (What time is it now?) was synthesized in reading style (default), to emphasize word "ji3dian3" (what time), and to emphasize "xian4zai4" (now). The input text mixed with the markup tags, if any, was in a form as follows.

- Reading style: xian4zai4ji3dian3le0?

- Emphasizing ji3dian3: < baseline $[-0.2,0]>$ xian4zai4 $<$ cap $[0.6]>$ ji3dian $3</$ cap $>$ le 0 ? $</$ baseline $>$

- Emphasizing xian4zai4: <baseline $[-0.2,0]><$ cap $[0.6]>$ xian4zai $4</$ cap $>$ ji3dian3le $0 ?</$ baseline $>$

The tag parameters, namely, $k=-0.2, b=0$, and $\zeta_{n}^{c}=0.6$, were determined by a preliminary experiment, and they were used to enhance emphasis in all the selected words.

It is observable from this example that the modulation is point by point in $F_{0}$, and the adjusting proportions can be specified by sparser targets. Point-by-point modulation makes it possible to rise and lower very local tones, and sparse specification of targets reduce the cost of predicting adjusting proportion $Z(t)$ in practice.

A small listening test was carried by three native Chinese speakers. There were 10 pairs of stimuli in total. The variant was the $F_{0}$ contours, while the others were common. The stimuli were heard in pairs: reading-prosody vs. its modified versions at a random order. The listeners were asked to point out which words were emphasized, or had "no difference."

In the listening test, $90 \%$ of samples with emphasis were identified. This and other informal listening confirmed the effectiveness of the frequency modulation in making emphasis in words. Though, the identified words did not match the target as well as we had expected. This is probably due to lacking necessary context for understanding the concept of "emphasis".

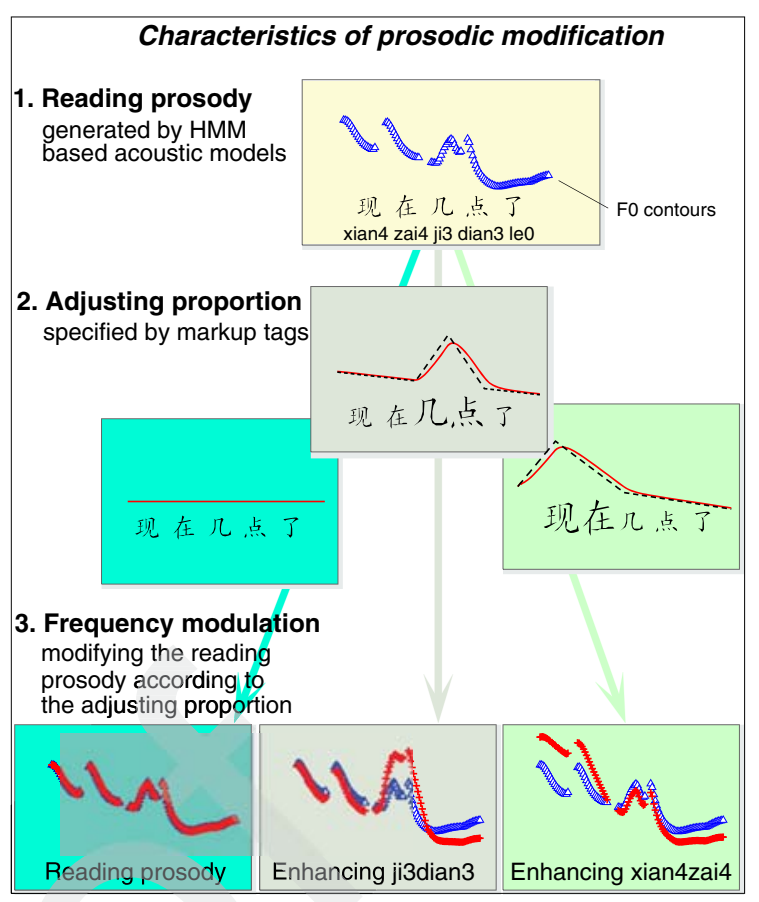

Figure 4: Illustration of enhancing emphasis in words with frequency modulation technique for rising and lowering tones.

\subsection{Experiment 2: Adding expressive dimensions}

This experiment was intended to test the significance of shifting frequency ranges and rescaling $F_{0}$ contours jointly. Shifting frequency ranges is one of ways of changing speaker's emotional states and speaking styles. In the previous work [7], our experimental results indicated that a speaker used quite different frequency ranges to communicate neutral news, good news and bad news, given the same writing prompts. In the case of conveying good news, for example, the frequency range went upward within the speaker's vocal range. On the other hand, the global movement of the $F_{0}$ contours in "good news" was declined less than those observed in reading speech, especially at the ending portion of the utterance.

We add some expressive dimensions to the reading prosody when conveying "good news" and "bad news" in a Japanese XIMERA voice with unit selection. For this purpose, we used ten ambiguous sentences as used in [7]. The sentences could be interpreted as good news, bad news, and neutral news. Five native speakers evaluated the appropriateness of synthetic speech in a listening test. The experimental setup is described below.

- Text-to-speech system:

- Voice: Japanese female.

- Synthesizer: Concatenation-based synthesis.

- Corpus size: 47 hours.

- Speaking style: Reading speech.

- The markup tags and their parameters used for shifting the frequency ranges and rescaling the $F_{0}$ contours:

- Neutral news: Plain text.

- Good news: <range [100,397, 131,420]> $<$ baseline $[0.1,-0.1]>$ the leading part of a sentence $<$ toend $[0.5]>$ the last accentual phrase of it $</$ toend $></$ baseline $></$ range $\rangle$. 


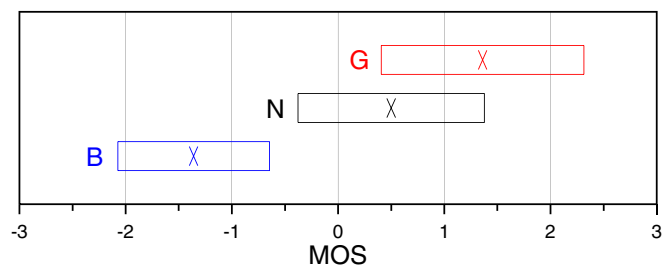

Figure 5: Mean opinion scores (the crosses) and standard deviations (the boxes) on a 7-point scale, - 3 (very good "bad news"), 0 (neutral), and +3 (very good "good news").

- Bad news: <range $[100,397,63,284]>$ $<$ baseline $[0,0.1]>$ text $</$ baseline $></$ range $>$.

- Listening test:

- Stimuli: $30(=10$ sentences $\times 3$ versions: bad news (B-version), neutral news (N-version), and good news (G-version)).

- Listeners: 5 native speakers.

- MOS: A 7-point scale, - 3 (very good "bad news"), 0 (neutral), and +3 (very good "good news").

The frequency ranges, i.e., [ $63 \mathrm{~Hz}$ and $284 \mathrm{~Hz}$ ] for bad news, [100 Hz, $397 \mathrm{~Hz}]$ for neutral news, and $[131 \mathrm{~Hz}$ and $420 \mathrm{~Hz}]$ for good news, were measured from around 3 hours of speech recorded by the speaker in each style. An upward scale was defined by a combination of baseline $(k=0.1$ and $b=-0.1)$ and toend $\left(\zeta_{n}^{e}=0.5\right)$ based on a preliminary experiment. The scale was used as the adjusting proportions $Z(t)$ to rescale the HMM-generated $F_{0}$ contours when synthesizing good news. There were three versions of synthetic speech for each sentence and 30 distinct stimuli in total used in the listening test. The listeners heard these stimuli in a random order over headphones in a silent office and evaluated the appropriateness of the synthetic speech at a 7-point scale as mentioned above.

The mean opinion scores (MOS) are shown in Fig. 5; N stands for the version synthesized by the HMM-generated $F_{0}$ contours; B for the version synthesized by rescaling the $F_{0}$ contours into the frequency ranges of bad news; and $\mathrm{G}$ for that of good news. The MOS are -1.36 (standard deviation (SD): 0.71 ) for B-version, 0.50 (SD: 0.87) for $\mathrm{N}$-version, and 1.36 (SD: 0.95) for G-version, respectively. Furthermore, we found that the listeners judged $94 \%$ of B-version samples as bad news (scores of $-1,-2$, and -3 ) and $78 \%$ of G-version samples as good news $(1,2$, and 3 ). Also, $30 \%$ of $\mathrm{N}$-version samples were judged as good news. The experimental results are very positive. They are comparable with those obtained by stylespecified corpora in the work of Sakai et al. where listeners identified the synthesized speech in a particular style between $98.4 \%$ for bad news and $66.7 \%$ for good news.

The prosodic modification was kept as the main variable in the evaluation experiments. Although the selected units probably were different from the bad news to the neutral news and the good news, they were selected from the same speech corpus that included 47 hours of reading speech. Therefore, the experimental results showed that the proposed method provides a sufficient means for modulating speaking tone, thus hopefully making synthetic speech more communicative.

\section{Discussion}

One of the advantages of the proposed frequency modulation technique is that the observed $F_{0}$ contours basically are used as they are; no extra parameter extraction is needed. In the conventional methods, for example, based on the Fujisaki model [10], it is necessary to extract the model parameters from the observed $F_{0}$ contours at first. However, automatic estimation of the model parameters still is a difficult task. While we have the powerful HMM technology [11], sufficiently labeled training data still is unavailable since expressive intonation is subtle. Prosodic modification shall be vital in advancing conventional/communicative speech synthesis. Also, it has other applications such as prosodic modification in a CALL (computerassisted language learning) system.

\section{Conclusion}

We presented a novel frequency modulation technique for prosody modification that can be developed as an extensional module of the conventional TTS systems. This technique provides a unified framework for representing the speaking tone (i.e., observed $F_{0}$ contours) and the adjusting proportions as well as manipulating the modulation of speaking tone according to the adjusting proportions. One of the advantages of this technique is that the observed $F_{0}$ contours basically are used as they are; no extra parameter extraction is needed. We evaluated this technique within the framework of XIMERA (a conventional TTS system) by using this technique to modify the readingstyle prosody, namely, enhancing emphasis in words and adding some expressive dimensions to them when conveying good and bad news. The experimental results indicated the effectiveness of this technique for adding subtle meaning to synthetic speech. However, it still is remained to see how to automatically design the adjusting proportions so as to map specific communication functions on to the observed $F_{0}$ contours. It shall be an attractive task in the future.

\section{References}

[1] K. Tokuda, et al., "Hidden Markov models based on multispace probability distribution for pitch pattern modling," in Proc. ICASSP, pp. 229-232, 1999.

[2] J. Pitrelli, et al., "The IBM expressive text-to-speech synthesis system for American English,” IEEE Trans. on Audio, Speech, and Lang. Processing, vol. 14, no. 4, 1109-1116, 2006.

[3] H. Kawai, et al., "XIMERA: A concatenative speech synthesis system with large scale corpora," IEICE Trans. Inf. \& Syst., vol. J89-D, no. 12, 2688-2698, 2006 (in Japanese).

[4] S. Nakamura, et al., "The ATR multi-lingual speech-to-speech translation system," IEEE Trans. on Speech and Audio Processing, vol. 14, no. 2, 365-376, 2006.

[5] Y. Sagisaka, T. Yamashita, and Y. Kokenawa, "Generation and perception of $F_{0}$ markedness for communicative speech synthesis," Speech Communication, 46, 376-384, 2005.

[6] N. Campbell, "Conversational speech synthesis and the need for some laughter," IEEE Trans. on Audio, Speech, and Lang. Processing, vol. 14, no. 4, 1171-1177, 2006.

[7] S. Sakai, et al., "Communicative speech synthesis with XIMERA: a first step," in SSW6-2007, 28-33, 2007.

[8] J. Ni, H. Kawai, and K. Hirose, "Constrained tone transformation technique for separation and combination of Mandarin tone and intonation,” J. Acoust. Soc. Am., 119(3):1764-1782, 2006.

[9] J. Ni and K. Hirose, "Quantitative and structural modeling of voice fundamental frequency contours of speech in Mandarin," Speech Communication, 48(8):989-1008, 2006.

[10] H. Fujisaki and K. Hirose, "Analysis of voice fundamental frequency contours for declarative sentences of Japanese," J. Acoust. Soc. Jpn (E), 5(4):233-242, 1984.

[11] http://hts.ics.nitech.ac.jp/ 